\title{
Spinocerebellar ataxia type 31 associated with REM sleep behavior disorder: a case report
}

\author{
Kazumasa Shindo ${ }^{*}$, Tohko Sato, Hiroaki Murata, Yuta Ichinose, Takanori Hata and Yoshihisa Takiyama
}

\begin{abstract}
Background: Spinocerebellar ataxia type 31 (SCA 31) is a slowly progressive neurodegenerative disorder characterized by pure cerebellar ataxia. Unlike other CAG repeat diseases, sleep-related problems have not been reported in patients with SCA 31 so far.
\end{abstract}

Case presentation: A 67-year-old woman was admitted to our hospital with dysarthria and gait disturbance after onset age of 62 years. Neurological examination revealed pure cerebellar ataxia. Genetic analysis detected expansion of a TGGAA repeat in the coding region of the BEAN/TK2 gene on chromosome 16p22.1, confirming the diagnosis of SCA 31. One year later, her husband noticed the patient talking loudly during sleep once or twice a week. Overnight polysomnography showed rapid eye movement sleep without atonia. Cardiac scintigraphy with iodine-123-labeled meta-iodobenzylguanidine revealed a low heart/mediastinum ratio, indicating reduced uptake, and a high washout rate.

Conclusion: To our knowledge, this is the first report of a patient with SCA 31 associated with rapid eye movement sleep behavior disorder (RBD). In the future, evaluation of autonomic function, assessment of the frequency of RBD, and performance of cardiac iodine-123-labeled meta-iodobenzylguanidine scintigraphy in a larger number of SCA 31 patients could be useful to resolve important issues regarding the mechanism of RBD.

Keywords: REM sleep behavior disorder, Spinocerebellar ataxia type 31, Polysomonography, Cardiac ${ }^{123}$ I-MIBG scintigraphy, a-Synucleinopathy

\section{Background}

Spinocerebellar ataxia 31 (SCA 31) is a slowly progressive neurodegenerative disorder characterized by onset in adulthood and pure cerebellar ataxia with or without nystagmus and hearing impairment $[1,2]$. Genetic analysis has revealed an insertion of TGGAA pentanucleotide repeats ranging in length from 2.5 to $3.8 \mathrm{~kb}$ between the brain expressed, associated with Nedd4 (BEAN) and the thymidine kinase 2 (TK2) gene and the gene located on chromosome 16p22.1 [3]. Among the various types of autosomal dominant cerebellar ataxia, SCA 31 is relatively common in the Japanese population [2], while it is rare in other East Asian countries such as Korea and

\footnotetext{
* Correspondence: kshindo@yamanashi.ac.jp

Department of Neurology, University of Yamanashi, 1110 Shimokatou, Yamanashi 409-3898, Japan
}

China [4, 5], and extremely rare among Caucasian populations [6].

Rapid eye movement (REM) behavior disorder (RBD) is a sleep disorder characterized by repetitive episodes of talking while asleep, calling out in a loud voice, sleep walking, and falling out of bed at night, while the affected person remains soundly asleep $[7,8]$. The diagnosis can be confirmed by lack of epileptic discharges on electroencephalography and by detecting REM sleep without atonia on polysomnography (PSG) [7]. Idiopathic RBD has been reported in $0.5-2 \%$ of healthy persons [8]. In addition, patients with neurodegenerative disorders, including $\alpha$-synucleinopathies such as Parkinson' s disease and dementia with Lewy body disease, frequently develop RBD during the period before and after the onset of motor symptoms $[9,10]$. Among the subtypes of SCA, almost a half of patients with Machado-Joseph disease (MJD) has been reported to

(c) The Author(s). 2019 Open Access This article is distributed under the terms of the Creative Commons Attribution 4.0 International License (http://creativecommons.org/licenses/by/4.0/), which permits unrestricted use, distribution, and 
develop RBD [11]. However, SCA 31 has not been associated with RBD to our knowledge, and the patient described here is the first case to be reported.

\section{Case presentation}

Written informed consent to this report was obtained from the patient. A 67-year-old Japanese woman was evaluated at the outpatient clinic of our hospital for slow progression of unsteadiness of gait/loss of balance and speech difficulty since five years earlier. She was taking two medicines (a calcium channel blocker for hypertension and a therapeutic agent for dyslipidemia) and using eye drops for glaucoma since before being hospitalized. The family history revealed that her mother had developed similar unsteadiness of gait and speech disturbance. At the initial assessment, neurological examination revealed no cognitive impairment. There was break-up of smooth pursuit eye movements, mild cerebellar dysarthria and limb ataxia, and a wide-based gait. She did not have sensory deficit nor extrapyramidal symptom. The score on the Scale for the Assessment and Rating of Ataxia was 11.5 points. The score on the Montreal Cognitive Assessment was $28 / 30$ points. Laboratory tests, chest X-ray film, electrocardiography, and echocardiography all gave normal results. Brain magnetic imaging showed mild cerebellar atrophy. Slight cerebellar hypoperfusion was observed on radioisotope scintigraphy of cerebral blood flow. Genetic analysis revealed expansion of a TGGAA repeat insertion in the coding region of the BEAN/YK2 gene on chromosome 16p22.1, confirming the diagnosis of SCA 31.

From one year later, her husband noticed her talking and calling out in a loud voice during sleep once or twice a week, while falling out of bed occurred once or twice per month. Because these episodes gradually became more frequent at age 72, she underwent PSG during sleep for suspected RBD. Electroencephalography revealed no epileptic discharges. PSG study demonstrated that total sleep time (TST) was $433.5 \mathrm{~min}$, REM sleep (\%TST) was $10.7 \%$, atonia index during REM sleep was 5.4 / hour, apnea-hypopnea index was 13.0 / hour (apnea index; 0.1 / hour), and periodic limb movements during sleep were 5.4 / hour. REM sleep without atonia was confirmed by PSG (Fig. 1). Evaluation of autonomic function showed loss of the sympathetic skin response related to sweating both at rest and with electrical stimulation, and a normal cutaneous vasomotor reflex response to electrical stimulation. Orthostatic hypotension was not evident, but she had occasional urinary incontinence. Dopamine transporter scintigraphy with ioflupane showed a normal finding that the mean specific binding ratio to the putamen/caudate was 4.84 (4.64 on the right side and 5.05 on the left side) (Fig. 2). Cardiac scintigraphy with iodine-123-labeled meta-iodobenzylguanidine $\left({ }^{123} \mathrm{I}-\mathrm{MIBG}\right)$ revealed a low heart/mediastinum ratio (early: 1.85; delayed: 1.32), indicating reduced uptake, and a high washout rate (74.4\%) (Fig. 3).

\section{Discussion and conclusions}

In this patient with SCA 31, recurrent episodes of talking and calling out loudly during sleep were noted six years after the onset of cerebellar ataxia and RBD was confirmed by detecting REM sleep without atonia on PSG. Previously, RBD has been reported in patients with various cerebellar degeneration syndromes $[8,10]$. Multiple system atrophy is generally associated with accumulation of $\alpha$-synuclein in the brain, and it has been reported that over $80 \%$ of these patients have RBD confirmed clinically or by PSG $[7,8]$. Among the various subtypes of SCA, MJD is most frequently associated with RBD [11], but patients with other subtypes of SCA (such

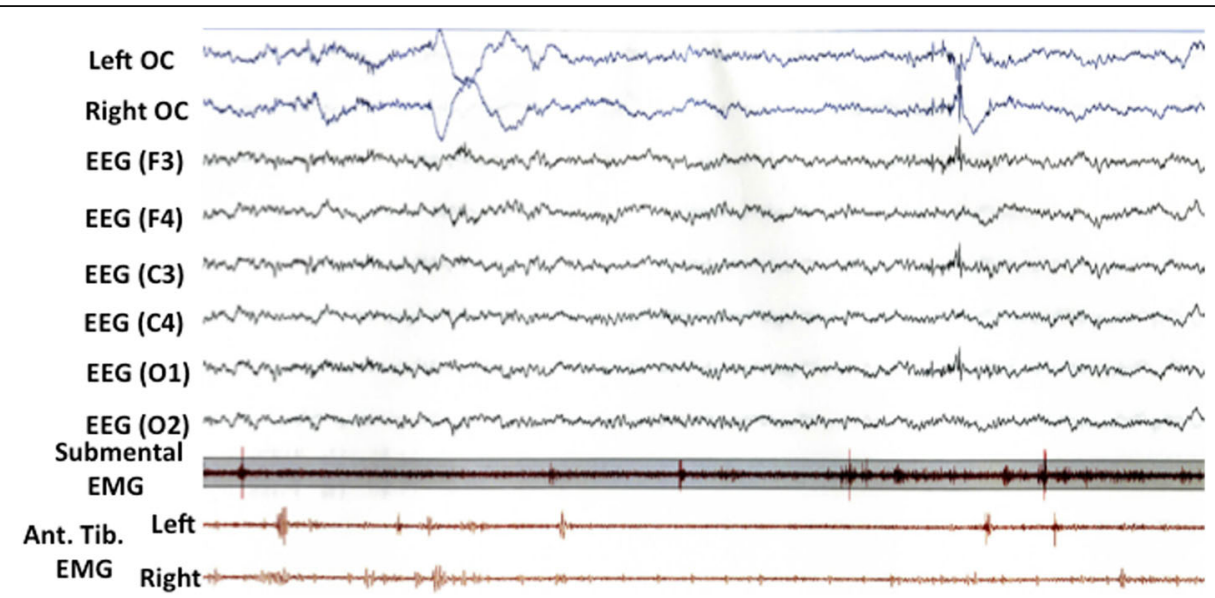

Fig. 1 The polysomnographic finding in this case. Excessive phasic/transient muscle activity confined to chin and anterior tibialis muscles (tenth and eleventh channels) was observed during rapid eye movement sleep. OC, oculogram; EEG, electroencephalogram; EMG, electromyogram, Ant. Tib., anterior tibial muscle 


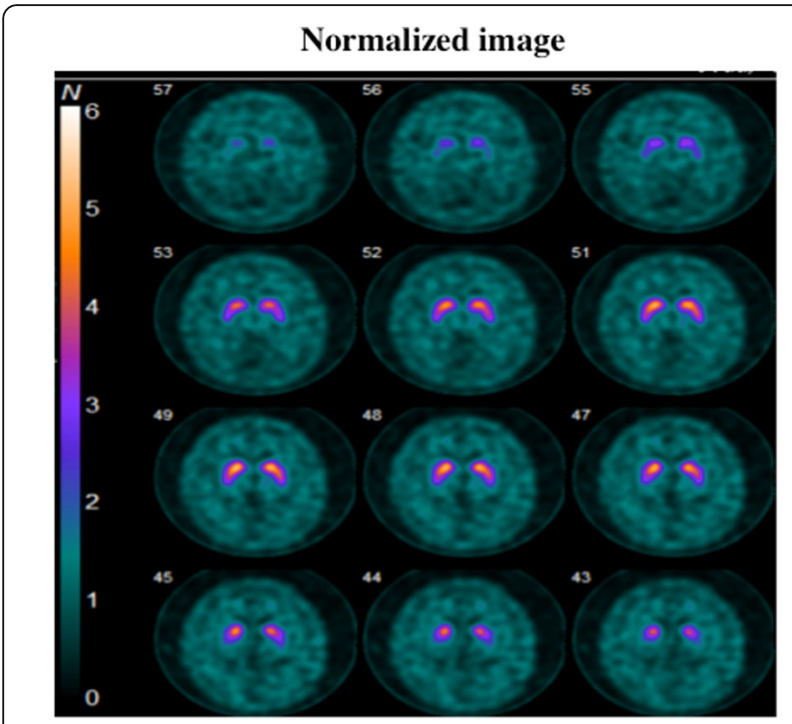

Specific binding ratio to the putamen/caudate: 4.84 (normal range; over 4.46 )

Fig. 2 Dopamine transporter scintigraphy with ioflupane in this case. The significant reduced uptake was not confirmed in putamen or caudate nucleus

as SCA 2 or DRPLA) sometimes have symptoms suggestive of RBD or PSG confirmation of RBD [12, 13]. However, there has been no previous report of RBD in a patient with SCA 31 as far as we could determine.

Patients with $\alpha$-synucleinopathies, including Parkinson's disease and dementia with Lewy bodies, frequently show reduced uptake of MIBG on cardiac scintigraphy
[14]. It has also been reported that patients with idiopathic RBD often have decreased MIBG uptake on cardiac scintigraphy $[7,8]$, and that many of them eventually develop an $\alpha$-synucleinopathy such as Parkinson's disease $[9,10]$. Therefore, one reason why RBD occurred in our patient could be a possible association with $\alpha$-synucleinopathy, since MIBG uptake was reduced on cardiac scintigraphy. However, involvement of the nigrostriatal dopaminergic network could not be confirmed by dopamine transporter scintigraphy.

Previous pathological studies and animal experiments have shown that complex impairment of several pontine nuclei (including the noradrenergic locus coeruleus and cholinergic nuclei), the substantia nigra, the hypothalamus, and the frontal cortex may be associated with the pathogenesis of RBD [7, 8]. Patients with MJD or SCA 2 often develop RBD, and brainstem dysfunction associated with progression of the pathologic process of SCA may lead to the onset of $\operatorname{RBD}[11,12]$. It is possible that RBD occurred in our patient due to pathologic changes associated with SCA 31. While pathological investigation of the brainstem has only revealed involvement of the inferior olivary nucleus in SCA 31 [15], it is still possible that sleep disorders like RBD arise from brainstem involvement, because pyramidal tract symptoms and involuntary movements suggesting brainstem lesions have been reported in SCA 31 patients [2]. Clinical detection of extrapyramidal symptoms or cognitive impairment would provide more information about the pathophysiological mechanism in the present case.

In our patient, cardiac scintigraphy revealed a decrease of MIBG uptake. As well as in patients with
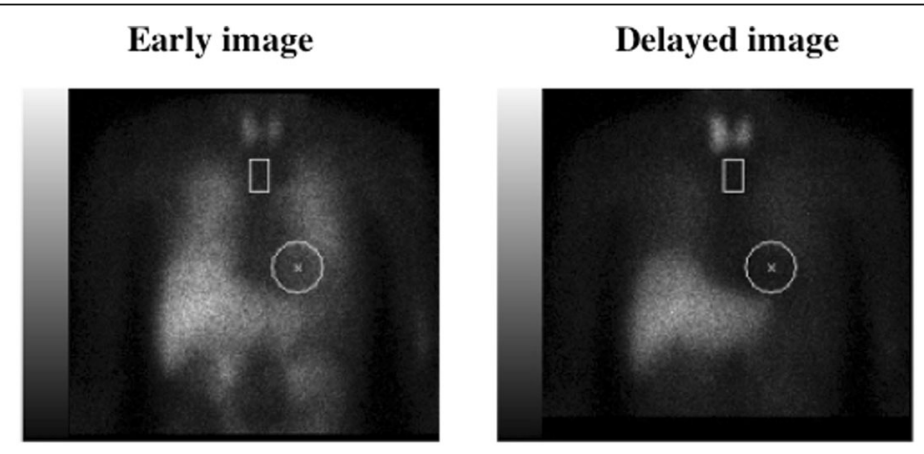
Heart
16.9 count/pixel Heart
6.5 count/pixel
Mediastinum
8.7 count/pixel Mediastinum
$4.8 \mathrm{count} / \mathrm{pixel}$

H/M: 1.85

H/M: 1.32

(normal range; over 2.2)

(normal range; over 2.2 )

Washout Rate: $\mathbf{7 4 . 4} \%$

(normal range; under $22 \%$ )

Fig. 3 The anterior planar images (early and delayed) of ${ }^{123} \mathrm{I}-\mathrm{MIBG}$ cardiac scintigraphy in this case. Cardiac MIBG uptake and $\mathrm{H} / \mathrm{M}$ ratio were reduced and washout rate was accelerated, compared to normal data. MIBG, meta-iodobenzylguanidine 
$\alpha$-synucleinopathies, this abnormality is often found in patients with diabetic autonomic neuropathy or familial amyloidotic polyneuropathy [16]. Thus, there may be subclinical autonomic involvement in SCA 31, because urinary incontinence and a reduced sweating response were noted in our patient and neurogenic bladder was reported in another patient with this disease [17]. There is the limitation in this study due to a single case report and since the possibility of the future complication of $\alpha$-synucleinopathy can not be denied, though current cognitive impairments or parkinsonian symptoms were not found and dopamine transporter scintigraphy with ioflupane showed a normal finding. In the future, evaluation of autonomic function, assessment of the frequency of RBD, and performance of cardiac MIBG scintigraphy in a larger number of SCA 31 patients could be useful to resolve important issues regarding the mechanism of RBD.

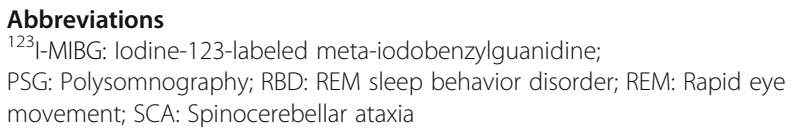

\section{Acknowledgements}

Not applicable.

\section{Funding}

All authors had no funding regarding this case report.

\section{Availability of data and meterials}

The data used and/or analyzed during the current study are available from the corresponding author on reasonable request.

\section{Authors' contributions}

KS and YT designed and organized the study. KS, TS and HM acquired the clinical and the laboratory data. KS, Yl, TH and YT carried out the molecular genetic studies and analyzed the molecular and the clinical data. KS wrote the manuscript that was edited by TS, HM, YI and TH. All authors have read and approved the final version of the manuscript submitted by KS.

\section{Ethics approval and consent to participate}

We confirm that we have read the journal's position on issues involved in ethical publication and affirm that this report is consistent with thouse guidelines. As this is a case report describing clinical observations, ethics approval was waived.

\section{Consent for publication}

Consent to publish was obtained in written form from the patient. Additionally, the patient consented to the publication of all personal and medical details included in the case report as well as the accompanying images.

\section{Competing interests}

The authors declare that they have no competing interests. None of the authors has any conflict of interest to disclose.

\section{Publisher's Note}

Springer Nature remains neutral with regard to jurisdictional claims in published maps and institutional affiliations.
Received: 6 September 2018 Accepted: 7 January 2019

Published online: 11 January 2019

\section{References}

1. Nagaoka U, Takashima M, Ishikawa K, Yoshizawa K, Yoshizawa T, Ishikawa M. A gene on SCA4 locus causes dominantly inherited pure cerebellar ataxia. Neurology. 2000;54:1971-5.

2. Nozaki H, Ikeuchi T, Kawakami A, Kimura A, Koide R, Tsuchiya M. Clinical and genetic characterizations of 16q-linked autosomal dominant spinocerebellar ataxia (AD-SCA) and frequency analysis of AD-SCA in the Japanese population. Mov Disord. 2007;22:857-62.

3. Sato N, Amino T, Kobayashi K, Asakawa S, Ishiguro T, Tsunemi T. Spinocerebellar ataxia type 31 is associated with "inserted" penta-nucleotide repeats containing (TGGAA)n. Am J Hum Genet. 2009;85:544-57.

4. Kim H-J, Jeon BS, Lee WY, Chung SJ, Yong SW, Kang JH. SCA in Korea and its regional distribution: a multicenter analysis. Parkinson Relat Disord. 2011; 17:72-5.

5. Outang Y, He Z, Li L, Qin X, Zhao Y, Yuan L. Spinocerebellar ataxia type 31 exists in Northeast China. J Neurol Sci. 2012;316:164-7.

6. Ishikawa K, Durr A, Klopstock T, Muller S, De Toffol B, Vidailhet M. Pentanucleotide repeats at the spinocerebellar ataxia type 31 (SCA 31) locus in Caucasians. Neurology. 201 1;77:1853-5.

7. Amulf I. REM sleep behavior disorder: motor manifestations and pathophysiology. Mov Disord. 2012;27:677-89.

8. St Louis EK, Boeve BF. REM sleep behavior disorder. Diagnosis, clinical implications, and future directions. Mayo Clin Proc. 2017:92:1723-36.

9. Schenck CH, Bundlie SR, Mahowald MW. Delayed emergence of a parkinsonian disorder in $38 \%$ of 29 older men initially diagnosed with idiopathic rapid eye movement sleep behavior disorder. Neurology. 1996;46: 388-93.

10. Postuma RB, Gagnon JF, Vendette M, Fantini ML, Massicotte-Marquecz J, Montplaisir J. Quantifying the risk of neurodegenerative disease in idiopathic REM sleep behavior disorder. Neurology. 2009;72:1296-300.

11. Silva GMF, Pedroso JL, Dos Santos DF, Braga-neto P, Do Prado LBF, De Carvalho LBC. NREM-related parasomnias in Machado-Joseph disease: clinical and polysomnographic evaluation. J Sleep Res. 2016;25:11-5.

12. Pedroso JL, Braga-neto P, Escorcio-Bezerra ML, Abrahao A, Cristino de Albuquerque MV. Non-motor and extracerebellar features in spinocerebellar ataxia type 2. Cerebellum. 2017;16:34-9.

13. Kim H, Yun JY, Choi K-G, Koo H, Han HJ. Sleep related problems as a nonmotor symptom of dentatorubropallidoluysian atrophy. J Krean Med Sci. 2018;33(17):e130.

14. Orimo M, Ozawa E, Nakade S, Sugimoto T, Mizusawa H. ${ }^{123}$ |metaiodobenzylguanidine myocardial scintigraphy in Parkinson's disease. J Neurol Neurosurg Psychiatry. 1999;67:189-94.

15. Adachi T, Kitayama M, Nakano T, Adachi Y, Kato S, Nakashima K. Autopsy case of spinocerebellar ataxia type 31 with severe dementia at the terminal stage. Neuropathology. 2015;35:273-9.

16. Orimo S, Yogo M, Nakamura T, Suzuki M, Watanabe H. ${ }^{123}$-metaiodobenzylguanidine (MIBG) cardiac scintigraphy in a-synucleinopathies. Ageing Res Rev. 2016;30:122-33.

17. Sugiyama M, Sakakibara R, Tateno F, Yano M, Takahashi O, Kishi M. Voiding dysfunction in spinocerebellar ataxia type 31. Kow Urin Tract Symptoms. 2014;6:64-7.

Ready to submit your research? Choose BMC and benefit from:

- fast, convenient online submission

- thorough peer review by experienced researchers in your field

- rapid publication on acceptance

- support for research data, including large and complex data types

- gold Open Access which fosters wider collaboration and increased citations

- maximum visibility for your research: over $100 \mathrm{M}$ website views per year

At BMC, research is always in progress.

Learn more biomedcentral.com/submission 